\title{
Efficacy of Neoadjuvant Single or Dual Anti-HER-2 Therapy Combined with Chemotherapy in Patients with HER-2-Positive Breast Cancer: A Single-Center Retrospective Study
}

\author{
Qian He, Jia-Yi Li, Qing-Lan Ren*
}

\begin{abstract}
Background: Studies have shown that neoadjuvant anti-HER-2 therapy and chemotherapy can increase pathologic complete response (pCR) rate in HER-2-positive breast cancer patients and improve prognosis. However, data from Chinese patients are limited. Therefore, we conducted a single-center retrospective study to evaluate the effects of neoadjuvant single or dual anti-HER-2 therapy and chemotherapy in Chinese HER-2-positive breast cancer patients and to explore the prognostic indicators of $\mathrm{pCR}$ and progression-free survival (PFS). Methods: We included patients with HER-2-positive breast cancer treated with neoadjuvant anti-HER-2 therapy and chemotherapy at the First Affiliated Hospital of Chongqing Medical University in China from January 2016 to July 2020. We analyzed the relationship between patient characteristics and the pCR rate or PFS. Results: Forty-seven patients with HER-2-positive breast cancer receiving neoadjuvant anti-HER-2 therapy and chemotherapy were included. Univariate analysis suggested that compared with patients receiving neoadjuvant single anti-HER-2 therapy, patients receiving neoadjuvant dual anti-HER-2 therapy tended to have a higher pCR rate and better PFS. Patients who achieved pCR also tended to have longer PFS. Multivariate analysis indicated that patients with greater systemic inflammation response index (SIRI) reduction $(>0.54)$ during neoadjuvant treatment (NAT) and patients with a lower T stage were more likely to achieve pCR. Patients aged $\leq 60$ years with lower Ki-67 had longer PFS. Conclusion: Greater SIRI reduction during NAT was an independent influencing factor for $\mathrm{pCR}$. Patients receiving neoadjuvant dual anti-HER-2 therapy and chemotherapy tended to have higher pCR rates and longer PFS. Patients who achieved pCR also tended to have longer PFS.
\end{abstract}

Keywords: Breast cancer- HER-2-positive- anti-HER-2 targeted therapy- pathologic complete response

Asian Pac J Cancer Prev, 22 (5), 1467-1475

\section{Introduction}

Breast cancer is the most common cancer in women and is one of the leading causes of cancer-related death in women (Siegel et al., 2019). With the development and application of anti-HER-2 drugs, the prognosis of breast cancer patients with HER-2 positivity has been significantly improved (Perez et al., 2011). Studies have found that the addition of targeted therapy to preoperative neoadjuvant therapy can improve the prognosis of HER-2-positive breast cancer patients without significantly increasing cardiac toxicity (Petrelli et al., 2011; Schneeweiss et al., 2013). The NeoSphere study found that in patients with HER-2-positive breast cancer, preoperative neoadjuvant chemotherapy (NAC) combined with dual anti-HER-2 therapy (trastuzumab and pertuzumab) significantly increased pathologic complete response (pCR) compared with chemotherapy combined with single anti-HER-2 therapy. Among patients who received preoperative anti-HER-2 therapy, those who achieved pCR had longer PFS than patients who did not achieve pCR (HR=0.54, 95\% CI: 0.29-1.00) (Gianni et al., 2016), and the cardiotoxicity of neoadjuvant dual anti-HER-2 therapy combined with chemotherapy was tolerable (Schneeweiss et al., 2013).

In addition to pCR, tumor staging, molecular subtype, histological characteristics, basic patient information, and treatment methods also have an impact on the prognosis of patients with HER-2-positive breast cancer (Ryu et al., 2017; Hwang et al., 2019). In addition, nutritional status can also influence the prognosis of cancer patients (Demark-Wahnefried et al., 2015). The prognostic nutritional index (PNI) is one of the most common parameters for assessing nutritional status. The PNI is equal to $10 \times$ serum albumin concentration $(\mathrm{g} / \mathrm{dL})$ $+0.005 \times$ total lymphocyte count $(/ \mathrm{mm} 3)$ (Mohri et al., 2016). Data have demonstrated that low PNI status before surgery or PNI reduction during NAC is a predictor of poor prognosis in many malignancies, including breast cancer (Tokunaga et al., 2015; Mohri et al., 2016; He et al., 2017; Migita et al., 2017; Hua et al., 2019; Oba 
et al., 2020). However, the role of PNI in breast cancer patients who received neoadjuvant anti-HER-2 therapy remains unclear. Some studies have also shown a link between cancer-related inflammation and treatment effects (Diakos et al., 2014). The systemic inflammation response index (SIRI: neutrophil count $\times$ monocyte count/ lymphocyte count) is based on neutrophils, monocytes and lymphocytes and can comprehensively evaluate the balance between immune and inflammatory states in patients. Evidence has shown that the SIRI can predict survival in some patients with malignant tumors (Qi et al., 2016; Li et al., 2017; Hua et al., 2020; Sun et al., 2020; Wang et al., 2020b). However, the prognostic value of the SIRI in neoadjuvant anti-HER-2 targeted therapy for breast cancer remains unclear.

However, much data exists on neoadjuvant anti-HER-2 therapy and chemotherapy in patients with HER-2-positive breast cancer in some countries (Hurvitz et al., 2018; Fasching et al., 2019). However, due to issues such as drug availability and medical insurance coverage, neoadjuvant anti-HER-2 therapy is still not widely used among Chinese patients with HER-2-positive breast cancer ( $\mathrm{Li}$ et al., 2018), especially neoadjuvant dual anti-HER-2 therapy. Therefore, this study aimed to explore the pCR rate of HER-2-positive breast cancer in Chinese patients receiving neoadjuvant anti-HER-2 therapy (including chemotherapy and trastuzumab and/or pertuzumab) and whether factors including PNI, SIRI, etc., could predict the $\mathrm{pCR}$ rate and postoperative PFS in patients with HER-2-positive breast cancer after receiving neoadjuvant anti-HER-2 therapy and chemotherapy.

\section{Materials and Methods}

\section{Patients and study design}

The search criteria included patients with HER-2-positive primary breast cancer using neoadjuvant anti-HER-2 therapy and chemotherapy at the First Affiliated Hospital of Chongqing Medical University in China from January 2016 to July 2020. The inclusion criteria were as follows: (1) females; (2) diagnosed with HER-2-positive invasive breast cancer (Wolff et al., 2014); (3) neoadjuvant therapy containing chemotherapy and trastuzumab and/ or pertuzumab; and (4) patients undergoing surgery after neoadjuvant treatment (5) whether or not to receive radiation and chemotherapy is determined according to the NCCN guidelines. The exclusion criteria were as follows: (1) distant metastasis at initial diagnosis; (2) patients who did not receive neoadjuvant treatment; (3) patients who did not receive surgery; and (4) no regular follow-up. Baseline characteristics and treatments were collected, including age, breast cancer staging, pathology, laboratory tests, imaging tests, treatment, surgery, survival and other data. The primary endpoint was PCR, and the secondary endpoint was PFS.

Neoadjuvant chemotherapy regimens included taxane-based regimens or anthracene-based regimens. Neoadjuvant anti-HER-2 therapy included trastuzumab (8 $\mathrm{mg} / \mathrm{kg}$ loading dose, then $6 \mathrm{mg} / \mathrm{kg}$, once every 3 weeks) and pertuzumab $\left(840 \mathrm{mg} / \mathrm{m}^{2}\right.$ loading dose, then $420 \mathrm{mg} / \mathrm{kg}$, once every 3 weeks). All patients underwent surgery. After surgery, all patients received anti-HER-2 treatment (except for a few patients who stopped targeting due to factors such as disease progression or the COVID-19 epidemic, a total of 1 year of anti-HER-2 treatment was received). The radiation oncologist decided whether to use adjuvant radiation. All hormone receptor-positive patients received adjuvant endocrine therapy. The patients were followed every 3 months.

PCR was defined as no residual invasive cancer cells in the breast or axillary lymph nodes (ypT0/is+ypN0) (von Minckwitz et al., 2012; Cortazar et al., 2014). Postoperative PFS was defined as the time from the start of the operation to tumor progression, death due to any reason or the last follow-up.

\section{Statistical analyses}

All statistical analyses were performed using Statistical Package for Social Sciences (SPSS, IBM Crop., Armok, NY, version 25) software. The $\chi 2$ test was used to determine the correlation between single/dual anti-HER-2 treatment and clinicopathological classification variables. Nonparametric tests were used to evaluate the pCR, 1-year PFS rate, 6-month PFS rate and 95\% confidence interval. Nonparametric tests were used to evaluate the pCR rate, disease progression rate and 95\% confidence interval. Pearson's $\chi^{2}$ test and Fisher's test were used to compare pCR, 1-year PFS, and 6-month PFS in patients from different groups. Univariate and multivariate binary logistic regression analyses were performed to analyze the association between $\mathrm{pCR}$ and clinicopathological variables. We reported odds ratios (ORs) and 95\% confidence intervals (CIs), and $\mathrm{P}<0.05$ was considered significant. The receiver operating characteristic (ROC) curve was used to determine the best clinical cut-off values of the PNI and SIRI for judging the postoperative survival status of patients, who were grouped accordingly. The Kaplan-Meier method and log-rank test were used to evaluate postoperative PFS. Univariate and multivariate Cox regression model analyses were used to determine the potential factors affecting postoperative PFS. We reported a hazard ratio (Mohri et al., 2016) and a 95\% confidence interval (95\% confidence interval), with significance defined as a $\mathrm{P}$ value $<0.05$.

\section{Results}

\section{Patient clinical and treatment characteristics}

Forty-seven patients with HER-2-positive breast cancer receiving neoadjuvant anti-HER-2 therapy and chemotherapy were included. Table 1 summarizes the characteristics of the included patients. The median age of the included patients was 52 years, and the median follow-up time was 10 months (calculated from the time of surgery). A total of 10 patients receiving neoadjuvant dual anti-HER-2 therapy and chemotherapy and 37 patients receiving neoadjuvant single anti-HER-2 therapy and chemotherapy were included. All patients underwent surgery and axillary lymph node dissection. All patients received anti-HER-2 therapy after surgery. Whether the patients underwent radiotherapy and/or endocrine therapy and/or chemotherapy was determined by the doctors. 
DOI: 10.31557/APJCP.2021.22.5.1467

Efficacy of Neoadjuvant Anti-HER-2 Therapy in Breast Cancer

Table 1. Patient, Disease and Treatment Characteristics

\begin{tabular}{|c|c|c|c|c|c|c|c|c|}
\hline \multirow[t]{2}{*}{ Variables } & & \multicolumn{2}{|c|}{ Total } & \multicolumn{2}{|c|}{ Single anti-HER-2 therapy } & \multicolumn{2}{|c|}{ Dual anti-HER-2 therapy } & \multirow[t]{2}{*}{$\mathrm{p}$} \\
\hline & & $\mathrm{n}$ & $(\%)$ & $\mathrm{n}$ & $(\%)$ & $\mathrm{n}$ & $(\%)$ & \\
\hline & & 47 & 100 & 37 & 78.7 & 10 & 21.3 & \\
\hline \multirow[t]{2}{*}{ Age } & $\leq 60$ & 40 & 85.1 & 31 & 77.5 & 9 & 22.5 & 1 \\
\hline & $>60$ & 7 & 14.9 & 6 & 85.7 & 1 & 14.3 & \\
\hline \multirow[t]{4}{*}{ T stage } & 1 & 8 & 17.0 & 5 & 62.5 & 3 & 37.5 & 0.465 \\
\hline & 2 & 23 & 48.9 & 18 & 78.3 & 5 & 21.7 & \\
\hline & 3 & 12 & 25.5 & 11 & 91.7 & 1 & 8.3 & \\
\hline & 4 & 1 & 2.1 & 1 & 100 & 0 & 0.0 & \\
\hline \multirow[t]{4}{*}{$\mathrm{N}$ stage } & 0 & 7 & 14.9 & 6 & 85.7 & 1 & 14.3 & 1 \\
\hline & 1 & 20 & 42.6 & 16 & 80.0 & 4 & 20.0 & \\
\hline & 2 & 4 & 8.5 & 4 & 100.0 & 0 & 0.0 & \\
\hline & 3 & 11 & 23.4 & 9 & 75.0 & 2 & 25.0 & \\
\hline \multirow[t]{2}{*}{$\mathrm{ER} \uparrow$} & negative & 30 & 63 & 25 & 83.3 & 5 & 16.7 & 0.512 \\
\hline & positive & 17 & 36.2 & 12 & 70.6 & 5 & 29.4 & \\
\hline \multirow[t]{2}{*}{$\mathrm{PR} \dagger$} & negative & 34 & 72.3 & 30 & 88.2 & 4 & 11.8 & 0.029 \\
\hline & positive & 13 & 27.7 & 7 & 53.8 & 6 & 46.2 & \\
\hline ER and $\mathrm{PR} \dagger$ & negative & 28 & 59.6 & 25 & 89.3 & 3 & 10.7 & 0.074 \\
\hline $\mathrm{ER}$ or $\mathrm{PR} \dagger$ & positive & 19 & 40.4 & 12 & 63.2 & 7 & 36.8 & \\
\hline \multirow[t]{2}{*}{ HER- $2 \uparrow$} & $2+$ & 9 & 19.1 & 5 & 55.6 & 4 & 44.4 & 0.151 \\
\hline & $3+$ & 38 & 80.9 & 32 & 84.2 & 6 & 15.8 & \\
\hline \multirow[t]{2}{*}{$\mathrm{Ki} 67 \dagger$} & $<30$ & 21 & 44.7 & 15 & 71.4 & 6 & 28.6 & 0.459 \\
\hline & $\geq 30$ & 26 & 55.3 & 22 & 84.6 & 4 & 15.4 & \\
\hline \multirow[t]{4}{*}{ pre-NAT PNI } & $\leq 54$ & 37 & 78.7 & 28 & 75.7 & 9 & 24.3 & 0.585 \\
\hline & $>54$ & 10 & 21.3 & 9 & 90.0 & 1 & 10.0 & \\
\hline & $\leq 53.3 \$$ & 20 & 57.1 & 16 & 80.0 & 4 & 20.0 & 0.68 \\
\hline & $>53.3 \ddagger$ & 15 & 42.9 & 13 & 86.7 & 2 & 13.3 & \\
\hline \multirow[t]{4}{*}{ post-NAT PNI } & $\leq 54$ & 40 & 85.1 & 31 & 77.5 & 9 & 22.5 & 0.558 \\
\hline & $>54$ & 3 & 6.4 & 2 & 66.7 & 1 & 33.2 & \\
\hline & $\leq 40.3 \ddagger$ & 2 & 5.7 & 1 & 50.0 & 1 & 50.0 & 0.335 \\
\hline & $>40.3 t$ & 31 & 88.6 & 26 & 83.9 & 5 & 16.1 & \\
\hline \multirow[t]{4}{*}{ pre-NAT PNI minus post-NAT PNI } & $\leq 9$ & 39 & 83 & 31 & 79.5 & 8 & 20.5 & 0.226 \\
\hline & $>9$ & 4 & 8.5 & 2 & 50.0 & 2 & 50.0 & \\
\hline & $\leq 4.875 t$ & 16 & 45.7 & 13 & 81.3 & 3 & 18.8 & 1 \\
\hline & $>4.875 \ddagger$ & 17 & 48.6 & 14 & 82.7 & 3 & 17.6 & \\
\hline \multirow[t]{4}{*}{ pre-NAT SIRI } & $\leq 0.785$ & 23 & 48.9 & 16 & 69.6 & 7 & 30.4 & 0.252 \\
\hline & $>0.785$ & 24 & 51.1 & 21 & 87.5 & 3 & 12.5 & \\
\hline & $\leq 0.465$ & 9 & 25.7 & 6 & 66.7 & 3 & 33.3 & 0.162 \\
\hline & $>0.465 \ddagger$ & 26 & 74.3 & 23 & 88.5 & 3 & 11.5 & \\
\hline \multirow[t]{4}{*}{ post-NAT SIRI } & $\leq 0.485$ & 13 & 27.7 & 7 & 53.8 & 6 & 46.2 & 0.039 \\
\hline & $>0.485$ & 32 & 68.1 & 28 & 87.5 & 4 & 12.5 & \\
\hline & $\leq 0.79 \ddagger$ & 14 & 40.0 & 9 & 64.3 & 5 & 35.7 & 0.061 \\
\hline & $>0.79 \ddagger$ & 20 & 57.1 & 19 & 95.0 & 1 & 5.0 & \\
\hline \multirow[t]{4}{*}{ pre-NAT SIRI minus post-NAT SIRI } & $\leq 0.54$ & 38 & 80.9 & 31 & 81.6 & 7 & 18.4 & 0.35 \\
\hline & $>0.54$ & 7 & 14.9 & 4 & 57.1 & 3 & 42.9 & \\
\hline & $\leq 1.905 \$$ & 32 & 91.4 & 26 & 81.3 & 6 & 18.8 & 1 \\
\hline & $>1.905 \ddagger$ & 1 & 2.9 & 1 & 100.0 & 0 & 0.0 & \\
\hline
\end{tabular}

ER, estrogen receptor; $n$, number; NAT, neoadjuvant treatment; PNI, prognostic nutritional index; PR, progesterone receptor; SIRI, systemic inflammation response index, $\%$ : percentage; $\uparrow$, before neoadjuvant treatment; $\ddagger$, in patients included in the PFS analysis ( $\mathrm{n}=35$ ). 


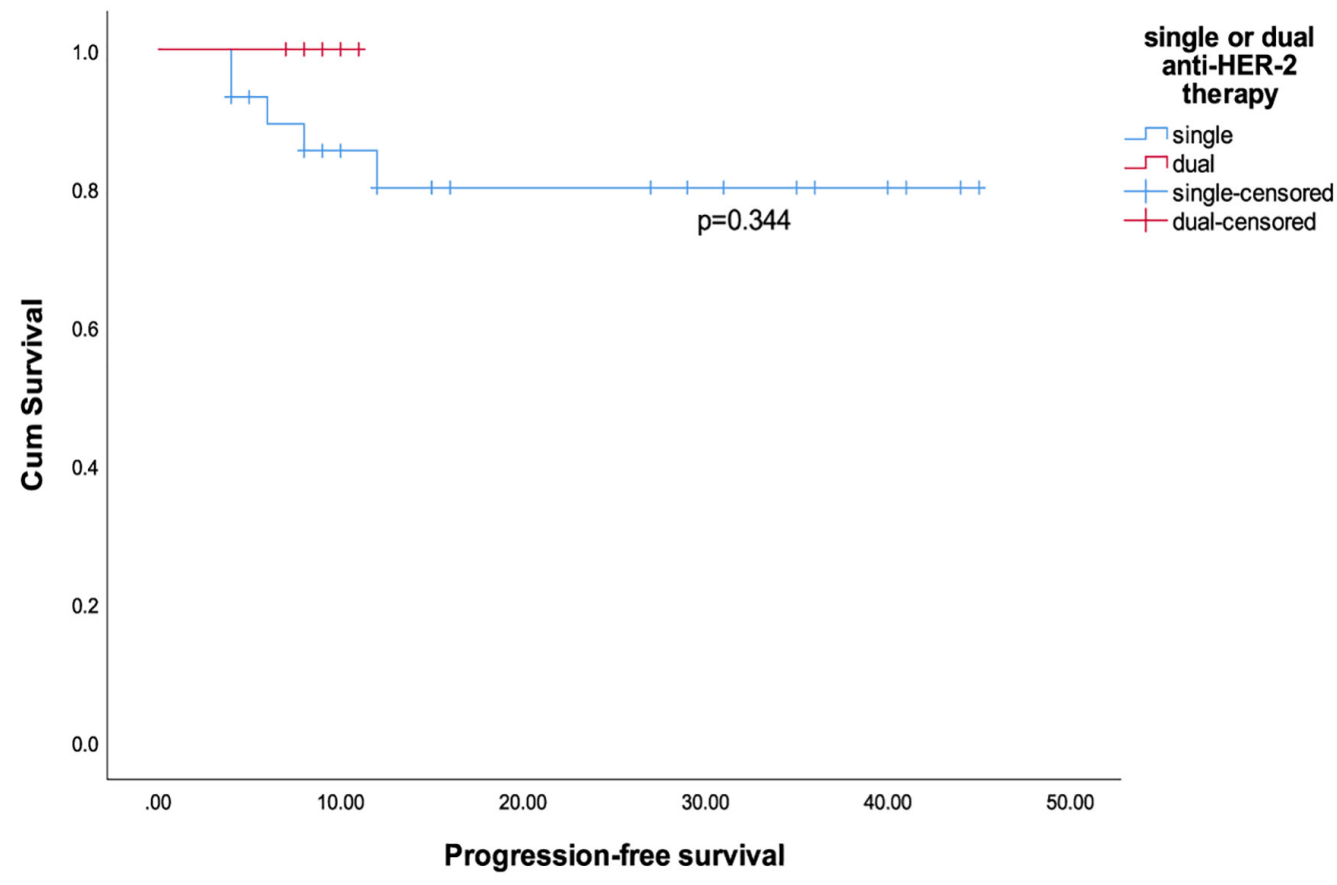

Figure 1. Kaplan-Meier Curves for Progression-Free Survival among Patients Receiving Neoadjuvant Single Anti-HER-2 Therapy and Chemotherapy and Patients Receiving Neoadjuvant Single anti-HER-2 Therapy and Chemotherapy.

Notably, two of the included patients relapsed. One relapsed 3 years after surgery, and one relapsed 12 years after surgery. Both patients were in disease-free survival after the operation.

\section{Pathologic complete response rates}

Among the 47 patients, 13 patients achieved pCR (27.7\%, 95\%CI:15.6\%-42.6\%). The pCR rates of patients receiving neoadjuvant single and dual anti-HER-2 treatment were $24.3 \%, 95 \% \mathrm{CI}: 11.8 \%-41.2 \%)$ and $40.0 \%$ (95\% CI: $12.2 \%-73.8 \%)(p=0.559)$. Univariate analysis suggested that compared with patients receiving neoadjuvant single anti-HER-2 therapy, patients receiving neoadjuvant dual anti-HER-2 therapy tended to achieve a higher $\mathrm{pCR}$ rate $(\mathrm{OR}=2.074, \mathrm{p}=0.331)$ (Table 2). Multivariate analysis suggested that patients whose SIRI decreased more $(>0.54)$ during NAT $(p=0.027)$ and patients with a lower T stage $(\mathrm{p}=0.016)$ were more likely to achieve pCR (Table 2).

\section{Progression-free survival}

We observed that the shortest progression time

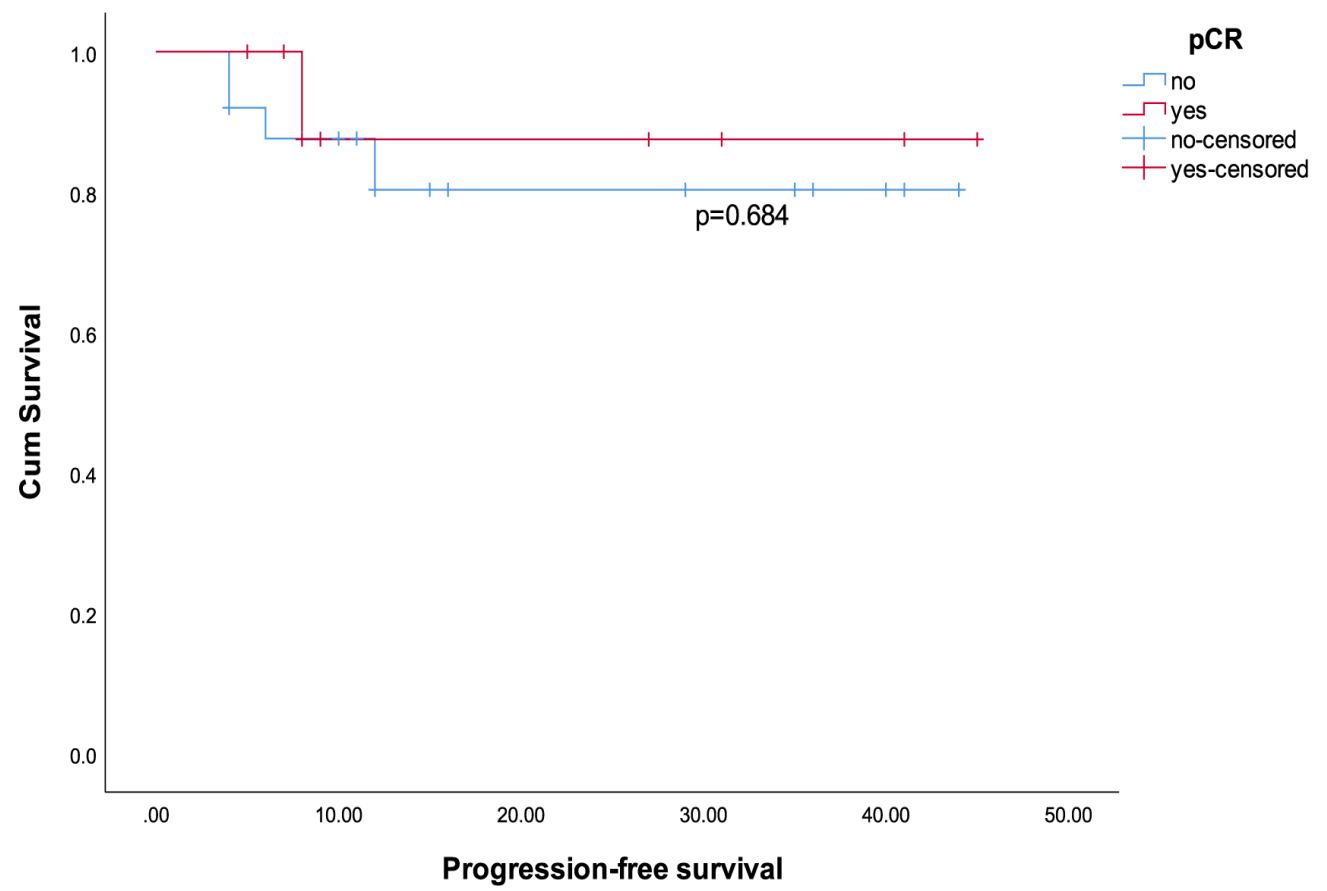

Figure 2. Kaplan-Meier Curves for Progression-Free Survival among Patients who Achieved pCR and Patients who did not Achieve pCR. 
DOI:10.31557/APJCP.2021.22.5.1467

Efficacy of Neoadjuvant Anti-HER-2 Therapy in Breast Cancer

Table 2. Pathologic Complete Response

\begin{tabular}{|c|c|c|c|c|c|c|c|}
\hline \multirow[t]{2}{*}{ Variables } & & \multicolumn{3}{|c|}{ Univariate analysis } & \multicolumn{3}{|c|}{ Multivariate analysis } \\
\hline & & $\mathrm{p}$ value & OR & $(95 \% \mathrm{CI})$ & $\mathrm{p}$ value & OR & $(95 \% \mathrm{CI})$ \\
\hline \multirow[t]{2}{*}{ age } & $\leq 60$ & ref & & & & & \\
\hline & $>60$ & 0.338 & 2.25 & $(0.428-11.824)$ & ref & & \\
\hline T stage & & 0.015 & 0.211 & $(0.061-0.737)$ & 0.016 & 0.016 & $(0.001-0.459)$ \\
\hline N stage & & 0.612 & 1.189 & $(0.609-2.322)$ & & & \\
\hline \multirow[t]{2}{*}{$\mathrm{ER}^{*}$} & negative & ref & & & & & \\
\hline & positive & 0.634 & 0.718 & $(0.183-2.814)$ & & & \\
\hline \multirow[t]{2}{*}{$\mathrm{PR}^{*}$} & negative & ref & & & & & \\
\hline & positive & 0.768 & 1.235 & $(0.304-5.020)$ & & & \\
\hline ER and PR* & negative & ref & & & & & \\
\hline ER or $\mathrm{PR}^{*}$ & positive & 0.865 & 0.893 & $(0.241-3.308)$ & & & \\
\hline \multirow[t]{2}{*}{ HER-2* } & $2+$ & ref & & & & & \\
\hline & $3+$ & 0.673 & 0.714 & $(0.150-3.408)$ & & & \\
\hline $\mathrm{Ki}-67^{*}$ & & 0.718 & 0.991 & $(0.945-1.040)$ & & & \\
\hline \multirow[t]{2}{*}{ Anti-HER-2 therapy } & single & ref & & & ref & & \\
\hline & dual & 0.331 & 2.074 & $(0.476-9.032)$ & 0.98 & 1.055 & $(0.017-64.358)$ \\
\hline \multirow[t]{2}{*}{ pre-NAT PNI } & $\leq 54$ & ref & & & ref & & \\
\hline & $>54$ & 0.085 & 3.625 & $(0.837-15.703)$ & 0.081 & 19.728 & $(0.693-561.605)$ \\
\hline \multirow[t]{2}{*}{ post-NAT PNI } & $\leq 54$ & ref & & & & & \\
\hline & $>54$ & 0.828 & 1.318 & $(0.108-16.039)$ & & & \\
\hline \multirow[t]{2}{*}{ pre-NAT PNI minus post-NAT PNI } & $\leq 9$ & ref & & & ref & & \\
\hline & $>9$ & 0.058 & 10 & $(0.923-108.331)$ & 0.576 & 0.253 & $(0.002-31.071)$ \\
\hline \multirow[t]{2}{*}{ pre-NAT SIRI } & $\leq 0.785$ & ref & & & ref & & \\
\hline & $>0.785$ & 0.131 & 2.85 & $(0.733-11.087)$ & 0.469 & 0.308 & $(0.013-7.457)$ \\
\hline \multirow[t]{2}{*}{ post-NAT SIRI } & $\leq 0.485$ & ref & & & & & \\
\hline & $>0.485$ & 0.585 & 1.515 & $(0.341-6.730)$ & & & \\
\hline \multirow{2}{*}{$\begin{array}{l}\text { pre-NAT SIRI minus post-NAT } \\
\text { SIRI }\end{array}$} & $\leq 0.54$ & ref & & & ref & & \\
\hline & $>0.54$ & 0.016 & 9.375 & $(1.525-57.621)$ & 0.027 & 1445.425 & $(2.322-899671.5)$ \\
\hline
\end{tabular}

CI, confidence interval; ER, estrogen receptor; NAT, neoadjuvant treatment; OR, odds ratio; PNI, prognostic nutritional index; PR, progesterone receptor; ref, reference; SIRI, systemic inflammation response index; *, before neoadjuvant treatment.

among all patients was 4 months, so we included patients with a follow-up period of $\geq 4$ months. We excluded 1 patient with incomplete survival data and 1 patient with primary lung cancer. A total of 35 patients were analyzed for PFS. The median follow-up time was 12 months, and the median PFS was 10 months. Among them, 20 patients were followed for more than 1 year (all were treated with neoadjuvant single anti-HER-2 therapy and chemotherapy), and the 1-year PFS rate was 75\% (95\% CI: $50.9 \%-91.3 \%$ ). All patients had a higher chance of recurrence within 6 months than after 6 months $(p=0.026)$. The 6-month PFS rates for all patients, patients receiving neoadjuvant dual anti-HER-2 therapy and chemotherapy, and patients receiving neoadjuvant single anti-HER-2 therapy and chemotherapy were $83.9 \%$ (95\% CI: $66.3 \%$ 94.5\%), 100\% (95\% CI: $54.1 \%-100 \%)$ and $80 \%(95 \%$ CI: $59.3 \%-93.2 \%)(\mathrm{p}=0.553)$, respectively. Patients who received neoadjuvant dual anti-HER-2 therapy and chemotherapy tended to have longer PFS than patients who received single anti-HER-2 therapy and chemotherapy (Figure 1, $\mathrm{p}=0.344$ ). By the end of follow-up, the average PFS of patients who achieved pCR with neoadjuvant therapy was 18.9 months (95\% CI: 7.79-30.01), while the average PFS of patients who did not achieve pCR was 16.16 months (95\% CI: 10.83-21.49) (Figure 2, $\mathrm{p}=0.684$ ). On univariate Cox regression, treatment with neoadjuvant dual anti-HER-2 and chemotherapy compared with single anti-HER-2 and chemotherapy tended to reduce the risk of disease progression $(\mathrm{HR}=0.037, \mathrm{p}=0.556)$. In addition, patients who achieved $\mathrm{pCR}(\mathrm{HR}=0.638, \mathrm{p}=0.688)$ also tended to obtain longer PFS (Table 3 ). On the multivariate Cox regression, patients aged $\leq 60$ years $(p=0.037)$, with lower Ki-67 ( $\mathrm{p}=0.050)$, and with greater SIRI reduction during NAT $(>1.905)(\mathrm{p}=0.026)$ had better PFS (Table 3 ). However, due to the large difference in the numbers of patients in the SIRI reduction high or low groups, the results may be biased.

\section{Discussion}

The purpose of this retrospective analysis was to evaluate the efficacy of neoadjuvant anti-HER-2 therapy combined with chemotherapy in unselected Chinese patients with HER-2-positive breast cancer. We found 
Table 3. Progression-Free Survival

\begin{tabular}{|c|c|c|c|c|c|c|c|}
\hline \multirow[t]{2}{*}{ Variable } & & \multicolumn{3}{|c|}{ Univariate Cox Regression } & \multicolumn{3}{|c|}{ Multivariate Cox Regression } \\
\hline & & $\mathrm{p}$ value & HR & $(95 \% \mathrm{CI})$ & $\mathrm{p}$ value & HR & $(95 \% \mathrm{CI})$ \\
\hline \multirow[t]{2}{*}{ age } & $\leq 60$ & ref & & & ref & & \\
\hline & $>60$ & 0.176 & 3.45 & $(0.574-20.745)$ & 0.037 & 134.484 & $(1.348-13418.243)$ \\
\hline T stage & & 0.158 & 2.213 & $(0.734-6.617)$ & 0.108 & 9.417 & $(0.611-145.129)$ \\
\hline $\mathrm{N}$ stage & & 0.917 & 0.954 & $(0.393-2.314)$ & & & \\
\hline \multirow[t]{2}{*}{$\mathrm{ER}^{*}$} & Negative & ref & & & & & \\
\hline & Positive & 0.338 & 0.342 & $(0.038-3.070)$ & & & \\
\hline \multirow[t]{2}{*}{$\mathrm{PR}^{*}$} & Negative & ref & & & & & \\
\hline & Positive & 0.814 & 0.766 & $(0.084-6.991)$ & & & \\
\hline ER and PR* & Negative & ref & & & & & \\
\hline ER or PR* & Positive & 0.292 & 0.307 & $(0.034-2.766)$ & & & \\
\hline \multirow[t]{2}{*}{ HER-2* } & $2+$ & ref & & & & & \\
\hline & $3+$ & 0.761 & 0.712 & $(0.079-6.392)$ & & & \\
\hline $\mathrm{Ki}-67^{*}$ & & 0.109 & 1.043 & (0.991-1.099) & 0.049 & 1.133 & $(1.001-1.283)$ \\
\hline \multirow[t]{2}{*}{ Anti-HER-2 therapy } & Single & ref & & & ref & & \\
\hline & Dual & 0.556 & 0.037 & $(0.0-2209.285)$ & 0.987 & 0.001 & $(0.0-0.0)$ \\
\hline \multirow[t]{2}{*}{ pre-NAT PNI } & $\leq 53.3$ & ref & & & & & \\
\hline & $>53.3$ & 0.468 & 1.941 & $(0.323-11.667)$ & & & \\
\hline \multirow[t]{2}{*}{ post-NAT PNI } & $\leq 40.3$ & ref & & & & & \\
\hline & $>40.3$ & 0.727 & 22.299 & $(0.0-84973360.8)$ & & & \\
\hline \multirow{2}{*}{$\begin{array}{l}\text { pre-NAT PNI minus } \\
\text { post-NAT PNI }\end{array}$} & $\leq 4.875$ & ref & & & & & \\
\hline & $>4.875$ & 0.303 & 68.79 & $(0.022-215861.8)$ & & & \\
\hline \multirow[t]{2}{*}{ pre-NAT SIRI } & $\leq 0.465$ & ref & & & & & \\
\hline & $>0.465$ & 0.453 & 30.413 & $(0.004-226078.6)$ & & & \\
\hline \multirow[t]{2}{*}{ post-NAT SIRI } & $\leq 0.79$ & ref & & & & & \\
\hline & $>0.79$ & 0.334 & 43.9 & $(0.020-94355.0)$ & & & \\
\hline \multirow{2}{*}{$\begin{array}{l}\text { pre-NAT SIRI minus } \\
\text { post-NAT SIRI }\end{array}$} & $\leq 1.905$ & ref & & & ref & & \\
\hline & $>1.905$ & 0.014 & 32 & $(2.002-511.602)$ & 0.026 & 198.665 & $(1.870-21110.073)$ \\
\hline \multirow[t]{2}{*}{$\mathrm{pCR}$} & No & ref & & & ref & & \\
\hline & Yes & 0.688 & 0.638 & $(0.071-5.715)$ & 0.931 & 1.128 & $(0.074-17.110)$ \\
\hline
\end{tabular}

CI, confidence interval; ER, estrogen receptor; HR, hazard ratio; NAT, neoadjuvant treatment; $p C R$, pathologic complete response; PNI, prognostic nutritional index; PR, progesterone receptor; ref, reference; SIRI, systemic inflammation response index; *, before neoadjuvant treatment.

that patients with a lower $\mathrm{T}$ stage were more likely to achieve pCR. Patients with advanced T stage of tumors, tended to have worse PFS. This was consistent with the results of other previous studies (Fayanju et al., 2018; Prat et al., 2020). In addition, patients aged $\leq 60$ years or with lower Ki-67 had better PFS. Other studies also showed that younger patients and patients with lower KI-67 were more likely to get longer PFS (Klauschen et al., 2015; Ignatiadis et al., 2019; Yu et al., 2019; Kanjanapan et al., 2020). The outcomes also showed that patients treated with neoadjuvant chemotherapy combined with dual anti-HER-2 therapy tended to obtain a higher pCR rate and better PFS than those treated with chemotherapy and single anti-HER-2 therapy. Patients who achieved pCR also tended to have a lower risk of disease progression. This was consistent with the results of the NeoSphere study. The NeoSphere study found that compared with patients receiving trastuzumab plus docetaxel, patients who were given pertuzumab and trastuzumab plus docetaxel had a significantly higher $\mathrm{pCR}$ rate with no significant difference in toxicity (Gianni et al., 2012). The 5-year follow-up results of this trial showed that patients with pCR had a longer PFS rate than those without pCR (5-year PFS rate was $85 \%$ for those with pCR and $76 \%$ for those without $\mathrm{pCR}, \mathrm{HR}=0.54,95 \% \mathrm{CI}: 0.291 .00)$ (Gianni et al., 2016). Similarly, the results of the Asian population subgroup analysis in the PEONY phase 3 randomized clinical trial also showed that compared with neoadjuvant trastuzumab and docetaxel, neoadjuvant pertuzumab and trastuzumab plus docetaxel therapy significantly increased the pCR rate of early or locally advanced HER-2-positive Asian breast cancer patients (Shao et al., 2020). Notably, in our study results, the $\mathrm{p}$ values for the $\mathrm{pCR}$ rate and PFS for single/dual anti-HER-2 therapy and the $p$ value of PFS for patients with pCR were both greater than 0.05 , indicating that there was no significant difference, which may be related to factors such as the small sample size of the included population, the insufficient follow-up 
time, and the timing of surgery. The National Surgical Adjuvant Breast and Bowel Project Protocols B-27 and its updated results indicated that patients with neoadjuvant chemotherapy who completed the total number of courses of adjuvant chemotherapy before surgery had a higher pCR rate than those who divided chemotherapy courses into preoperative chemotherapy and postoperative chemotherapy, while patients who achieved pCR had better disease-free survival (DFS) and overall survival (OS) (Bear et al., 2003; Rastogi et al., 2008). However, due to the patients' will and the limitations of the patients' economic conditions and the medical environment in China, for some chemotherapy regimens that require the completion of 8 cycles before surgery, there were cases in China in which surgery was performed in advance after the completion of 4 cycles, and the remaining 4 cycles of chemotherapy were completed after surgery. Not all patients who did not achieve pCR underwent further chemotherapy after surgery. This could affect the results of the study. We will continue to follow the prognosis of patients to further determine the optimal timing of surgery. Larger studies are needed to further evaluate the efficacy of neoadjuvant anti-HER-2 therapy combined with chemotherapy in Chinese patients with HER-2-positive breast cancer and the selection of surgical timing for patients receiving neoadjuvant chemotherapy combined with anti-HER-2 treatment.

Numerous studies have shown that tumors are associated with systemic inflammation (Romero-Cordoba et al., 2019; Tuomisto et al., 2019; Fest et al., 2020). As a simple and easily available prognostic indicator, the SIRI shows certain prognostic value in many tumors (including breast cancer) (Qi et al., 2016; Sun et al., 2020). Our study suggested that patients with greater SIRI reduction $(>0.54)$ during neoadjuvant therapy had a higher $\mathrm{pCR}$ rate $(\mathrm{p}=$ 0.027). Similarly, some studies showed that in esophageal cancer and cervical cancer, patients whose SIRI decreased more after surgery may have a better prognosis (Geng et al., 2018; Chao et al., 2020). Hua et al. found that a lower SIRI $(<0.54)$ was a good prognostic factor for postmenopausal breast cancer patients $(\mathrm{p}=0.008)$ (Hua et al., 2020). Chen et al. found that a lower SIRI $(<0.85)$ in breast cancer patients before NAC indicated better DFS and OS ( $p=0.011, p=0.017)($ Chen et al., 2020). Wang et al. found that the OS rate of breast cancer patients with an SIRI $<0.65$ before or after surgery was significantly higher than that of breast cancer patients with an SIRI $>0.65$ $(p<0.001)$ (Wang et al., 2020b). Although our study did not show that the more decrease in SIRI during neoadjuvant therapy was associated with longer PFS. This may because patients may have drug-induced leukopenia while receiving neoadjuvant therapy, and the overuse of drugs that raises leukocytes could affect SIRI. As the treatment becomes more and more standardized, we will continue to follow up more patients to obtain more accurate results in the future. The possible reason that the SIRI affects the prognosis of patients is that neutrophils can promote tumor proliferation and metastasis and can release some cytokines and chemokines to suppress the immune system (Laviron et al., 2019). Monocytes, especially TAMs, have an impact on the tumor microenvironment by promoting

\section{Efficacy of Neoadjuvant Anti-HER-2 Therapy in Breast Cancer}

tumor progression and metastasis (Gregory and Houghton, 2011). Lymphocytes are an important part of antitumor immunity and immune surveillance and inhibit tumor cell proliferation and migration by inducing cytotoxic cell death (Mantovani et al., 2008). Therefore, a lower SIRI might predict better outcomes for breast cancer patients. More clinical data are still needed to verify this view.

Many studies found that a high PNI was an independent positive prognostic factor in many tumors (such as cancer and lung cancer) (Tokunaga et al., 2015; Nakatani et al., 2017; Jin et al., 2018; Wang et al., 2020a). In breast cancer, Mohri et al. also found that patients with high preoperative PNI status had a better 5 -year OS rate $(\mathrm{p}=0.013)$ (Mohri et al., 2016). Oba et al., (2020) found that excessive PNI reduction during NAC in breast cancer patients $(>5.26)$ was related to poor prognosis in breast cancer, suggesting that breast cancer patients who maintain a better nutritional status during NAC may obtain better effects. The univariate analysis in our results suggested that patients with a higher PNI before neoadjuvant therapy or surgery tended to have a higher pCR rate and longer PFS, but these results were not statistically significant. This may be due to the limited sample size included. More studies are needed to evaluate the significance of the PNI in the treatment of breast cancer patients with neoadjuvant chemotherapy combined with anti-HER-2 drugs.

Accurately predicting the prognosis of breast cancer patients is of great significance. It can help clinicians develop more appropriate treatment plans. Our study is a preliminary evaluation of the efficacy of neoadjuvant antiHER-2 therapy and chemotherapy in Chinese patients with HER-2-positive breast cancer, and more studies are needed to comprehensively evaluate the prognosis of patients.

\section{Limitations}

1) Due to the availability of drugs, patient economic conditions, and medical insurance coverage, breast cancer patients in our center have only gradually begun to use anti-HER-2 drugs in recent years, especially dual-anti-HER-2 therapy. The time that these drugs were used for neoadjuvant therapy was even shorter. As a single-center retrospective study, the sample size was small. The follow-up time was relatively short. These may lead to biased results. The small sample size may make certain factors that enable patients to obtain higher pCR rates and longer PFS only show corresponding trends in this study, and may also cause the interference effects of confounding factors to be amplified. In addition, many patients had no tumor progression at the time of follow-up, so the exact time of PFS was not available. By the end of follow-up, only 1 patient died, so we could not assess the OS. We would expand the sample size in the future and continue to follow up to further verify our results. More prospective studies are needed to evaluate the efficacy of neoadjuvant anti-HER-2 therapy and chemotherapy in Chinese patients with HER-2-positive breast cancer.

2) The population we included may have a combination of different neoadjuvant chemotherapy regimens, and some Chinese breast cancer patients started surgery early after completing only 4 cycles of treatment, which may influence the results. And due to poor recovery of hand 
function after surgery, some patients may not be able to receive radiotherapy on time. This may also affect the patient's OS. Further studies are needed to evaluate the effects of the neoadjuvant anti-HER-2 combined chemotherapy regimen and surgical timing on the chemotherapy response and prognosis of breast cancer patients in China.

3) As a retrospective study, it has some inherent limitations. The outcomes were hypothesis generating and showed that patients with greater SIRI reduction $(>0.54)$ during neoadjuvant therapy and patients with a lower $\mathrm{T}$ stage had higher $\mathrm{pCR}$ rates. Patients who were $\leq 60$ years old, had lower Ki-67 had longer PFS. Patients receiving neoadjuvant dual anti-HER-2 therapy and chemotherapy tended to have a higher $\mathrm{pCR}$ rate and better PFS. Patients with pCR also tended to obtain better PFS.

In conclusion, the greater reduction in SIRI during NAT indicated the greater possibility of achieve pCR. Patients receiving neoadjuvant dual anti-HER-2 therapy and chemotherapy had a tendency to reach pCR and had a longer PFS. It is very important to accurately predict the patient's prognosis, which helps make clinical decisions. We will continue to follow up more patients to verify our hypothesis.

\section{Author Contribution Statement}

(I) Conception and design: All authors; (II) Administrative support: None; (III) Provision of study materials or patients: QH, QR; (IV) Collection and assembly of data: QH, JL; (V) Data analysis and interpretation: QH; (Perez et al.) Manuscript writing: All authors; (VII) Final approval of manuscript: All authors.

\section{Acknowledgments}

\section{Ethical Approval}

Due to the use of de-identified data, the study was exempted by the institutional review board.

\section{Conflict of interest}

The authors have no conflicts of interest to declare.

\section{References}

Bear HD, Anderson S, Brown A, et al (2003). The effect on tumor response of adding sequential preoperative docetaxel to preoperative doxorubicin and cyclophosphamide: preliminary results from National Surgical Adjuvant Breast and Bowel Project Protocol B-27. J Clin Oncol, 21, 4165-74.

Chao B, Ju X, Zhang L, et al (2020). A novel prognostic marker systemic inflammation response index (SIRI) for operable cervical cancer patients. Front Oncol, 10, 766.

Chen L, Kong X, Wang Z, et al (2020). Pretreatment systemic inflammation response index in patients with breast cancer treated with neoadjuvant chemotherapy as a useful prognostic indicator. Cancer Manag Res, 12, 1543-67.

Cortazar P, Zhang L, Untch M, et al (2014). Pathological complete response and long-term clinical benefit in breast cancer: the CTNeoBC pooled analysis. Lancet, 384, 164-72.

Demark-Wahnefried W, Rogers LQ, Alfano CM, et al (2015). Practical clinical interventions for diet, physical activity, and weight control in cancer survivors. CA Cancer J Clin, 65, 167-89.

Diakos CI, Charles KA, McMillan DC, et al (2014). Cancer-related inflammation and treatment effectiveness. Lancet Oncol, 15, e493-503.

Fasching PA, Hartkopf AD, Gass P, et al (2019). Efficacy of neoadjuvant pertuzumab in addition to chemotherapy and trastuzumab in routine clinical treatment of patients with primary breast cancer: a multicentric analysis. Breast Cancer Res Treat, 173, 319-28.

Fayanju OM, Ren Y, Thomas SM, et al (2018). The clinical significance of breast-only and node-only pathologic complete response (pCR) after neoadjuvant chemotherapy (NACT): A Review of 20,000 Breast Cancer Patients in the National Cancer Data Base (NCDB). Ann Surg, 268, 591-601.

Fest J, Ruiter R, Mulder M, et al (2020). The systemic immuneinflammation index is associated with an increased risk of incident cancer-A population-based cohort study. Int $J$ Cancer, 146, 692-8.

Geng Y, Zhu D, Wu C, et al (2018). A novel systemic inflammation response index (SIRI) for predicting postoperative survival of patients with esophageal squamous cell carcinoma. Int Immunopharmacol, 65, 503-10.

Gianni L, Pienkowski T, Im YH, et al (2012). Efficacy and safety of neoadjuvant pertuzumab and trastuzumab in women with locally advanced, inflammatory, or early HER2-positive breast cancer (NeoSphere): a randomised multicentre, open-label, phase 2 trial. Lancet Oncol, 13, 25-32.

Gianni L, Pienkowski T, Im YH, et al (2016). 5-year analysis of neoadjuvant pertuzumab and trastuzumab in patients with locally advanced, inflammatory, or early-stage HER2-positive breast cancer (NeoSphere): a multicentre, open-label, phase 2 randomised trial. Lancet Oncol, 17, 791-800.

Gregory AD, Houghton AM (2011). Tumor-associated neutrophils: new targets for cancer therapy. Cancer Res, 71, 2411-6.

He ZQ, Ke C, Al-Nahari F, et al (2017). Low preoperative prognostic nutritional index predicts poor survival in patients with newly diagnosed high-grade gliomas. J Neurooncol, 132, 239-47.

Hua X, Long ZQ, Huang X, et al (2019). The value of prognostic nutritional index (PNI) in predicting survival and guiding radiotherapy of patients with T1-2N1 breast cancer. Front Oncol, 9, 1562.

Hua X, Long ZQ, Huang X, et al (2020). The preoperative systemic inflammation response index (SIRI) independently predicts survival in postmenopausal women with breast cancer. Curr Probl Cancer, 44, 100560.

Hurvitz SA, Martin M, Symmans WF, et al (2018). Neoadjuvant trastuzumab, pertuzumab, and chemotherapy versus trastuzumab emtansine plus pertuzumab in patients with HER2-positive breast cancer (KRISTINE): a randomised, open-label, multicentre, phase 3 trial. Lancet Oncol, 19, 115-26.

Hwang KT, Kim J, Jung J, et al (2019). Impact of breast cancer subtypes on prognosis of women with operable invasive breast cancer: A Population-based Study Using SEER Database. Clin Cancer Res, 25, 1970-9.

Ignatiadis M, Van den Eynden G, Roberto S, et al (2019). Tumorinfiltrating lymphocytes in patients receiving trastuzumab/ pertuzumab-based chemotherapy: A TRYPHAENA Substudy. J Natl Cancer Inst, 111, 69-77.

Jin S, Cao S, Xu S, et al (2018). Clinical impact of pretreatment prognostic nutritional index (PNI) in small cell lung cancer patients treated with platinum-based chemotherapy. Clin 
Respir J, 12, 2433-40.

Kanjanapan Y, Lok SW, Gibbs P, et al (2020). Impact of prior (neo)adjuvant trastuzumab (NAT) exposure on the efficacy of HER2-targeted therapy for metastatic breast cancer. Breast Cancer Res Treat, 184, 87-95.

Klauschen F, Wienert S, Schmitt WD, et al (2015). Standardized Ki67 diagnostics using automated scoring--clinical validation in the gepartrio breast cancer study. Clin Cancer Res, 21, 3651-7.

Laviron M, Combadière C, Boissonnas A (2019). Tracking monocytes and macrophages in tumors with live imaging. Front Immunol, 10, 1201.

Li J, Shao Z, Xu B, et al (2018). Use of trastuzumab as an adjuvant/neoadjuvant therapy in patients with HER2-positive breast cancer in China: The Nvwa study. Medicine (Baltimore), 97, e10350.

Li S, Lan X, Gao H, et al (2017). Systemic Inflammation Response Index (SIRI), cancer stem cells and survival of localised gastric adenocarcinoma after curative resection. J Cancer Res Clin Oncol, 143, 2455-68.

Mantovani A, Allavena P, Sica A, et al (2008). Cancer-related inflammation. Nature, 454, 436-44.

Migita K, Matsumoto S, Wakatsuki K, et al (2017). A decrease in the prognostic nutritional index is associated with a worse long-term outcome in gastric cancer patients undergoing neoadjuvant chemotherapy. Surg Today, 47, 1018-26.

Mohri T, Mohri Y, Shigemori T, et al (2016). Impact of prognostic nutritional index on long-term outcomes in patients with breast cancer. World J Surg Oncol, 14, 170.

Nakatani M, Migita K, Matsumoto S, et al (2017). Prognostic significance of the prognostic nutritional index in esophageal cancer patients undergoing neoadjuvant chemotherapy. Dis Esophagus, 30, 1-7.

Oba T, Maeno K, Takekoshi D, et al (2020). Neoadjuvant chemotherapy-induced decrease of prognostic nutrition index predicts poor prognosis in patients with breast cancer. BMC Cancer, 20, 160.

Perez EA, Romond EH, Suman VJ, et al (2011). Four-year follow-up of trastuzumab plus adjuvant chemotherapy for operable human epidermal growth factor receptor 2-positive breast cancer: joint analysis of data from NCCTG N9831 and NSABP B-31. J Clin Oncol, 29, 3366-73.

Petrelli F, Borgonovo K, Cabiddu M, et al (2011). Neoadjuvant chemotherapy and concomitant trastuzumab in breast cancer: a pooled analysis of two randomized trials. Anticancer Drugs, 22, 128-35.

Prat A, Pascual T, De Angelis C, et al (2020). HER2-enriched subtype and ERBB2 expression in HER2-positive breast cancer treated with dual HER2 blockade. J Natl Cancer Inst, 112, 46-54.

Qi Q, Zhuang L, Shen Y, et al (2016). A novel systemic inflammation response index (SIRI) for predicting the survival of patients with pancreatic cancer after chemotherapy. Cancer, 122, 2158-67.

Rastogi P, Anderson SJ, Bear HD, et al (2008). Preoperative chemotherapy: updates of national surgical adjuvant breast and bowel project protocols B-18 and B-27. J Clin Oncol, 26, 778-85.

Romero-Cordoba S, Meneghini E, Sant M, et al (2019). Decoding immune heterogeneity of triple negative breast cancer and its association with systemic inflammation. Cancers (Basel), 11.

Ryu JM, Yu J, Kim SI, et al (2017). Different prognosis of young breast cancer patients in their 20s and 30s depending on subtype: a nationwide study from the Korean Breast Cancer Society. Breast Cancer Res Treat, 166, 833-42.

Schneeweiss A, Chia S, Hickish T, et al (2013). Pertuzumab
Efficacy of Neoadjuvant Anti-HER-2 Therapy in Breast Cancer

plus trastuzumab in combination with standard neoadjuvant anthracycline-containing and anthracycline-free chemotherapy regimens in patients with HER2-positive early breast cancer: a randomized phase II cardiac safety study (TRYPHAENA). Ann Oncol, 24, 2278-84.

Shao Z, Pang D, Yang H, et al (2020). Efficacy, safety, and tolerability of pertuzumab, trastuzumab, and docetaxel for patients with early or locally advanced ERBB2-positive breast cancer in Asia: The PEONY Phase 3 Randomized Clinical Trial. JAMA Oncol, 6, e193692.

Siegel RL, Miller KD, Jemal A (2019). Cancer statistics, 2019. CA Cancer J Clin, 69, 7-34.

Sun L, Hu W, Liu M, et al (2020). High systemic inflammation response index (SIRI) indicates poor outcome in gallbladder cancer patients with surgical resection: A Single Institution Experience in China. Cancer Res Treat, 52, 1199-210.

Tokunaga R, Sakamoto Y, Nakagawa S, et al (2015). Prognostic nutritional index predictssevere complications, recurrence, and poor prognosis in patients with colorectal cancer undergoing primary tumor resection. Dis Colon Rectum, 58, 1048-57.

Tuomisto AE, Mäkinen MJ, Väyrynen JP (2019). Systemic inflammation in colorectal cancer: Underlying factors, effects, and prognostic significance. World J Gastroenterol, 25, 4383-404.

von Minckwitz G, Untch M, Blohmer JU, et al (2012). Definition and impact of pathologic complete response on prognosis after neoadjuvant chemotherapy in various intrinsic breast cancer subtypes. J Clin Oncol, 30, 1796-804.

Wang J, Liu Y, Mi X, et al (2020a). The prognostic value of prognostic nutritional index (PNI) and neutrophil to lymphocyte ratio (NLR) for advanced non-small cell lung cancer treated with platinum-based chemotherapeutics. Ann Palliat Med, 9, 967-78.

Wang L, Zhou Y, Xia S, et al (2020b). Prognostic value of the systemic inflammation response index (SIRI) before and after surgery in operable breast cancer patients. Cancer Biomark, 28, 537-47.

Wolff AC, Hammond ME, Hicks DG, et al (2014). Recommendations for human epidermal growth factor receptor 2 testing in breast cancer: American Society of Clinical Oncology/College of American Pathologists clinical practice guideline update. Arch Pathol Lab Med, 138, 241-56.

Yu KD, Wu SY, Liu GY, et al (2019). Concurrent neoadjuvant chemotherapy and estrogen deprivation in patients with estrogen receptor-positive, human epidermal growth factor receptor 2-negative breast cancer (CBCSG-036): A randomized, controlled, multicenter trial. Cancer, 125, 2185-93.

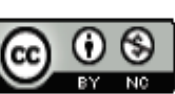

This work is licensed under a Creative Commons AttributionNon Commercial 4.0 International License. 